\title{
Tuberculosis pulmonar cavitaria una presentación inusual en pediatría
}

\author{
DOI: $10.5377 /$ alerta.v4i3.10907 \\ Escobar $^{3}$ \\ 1, 2 y 3. Hospital Nacional de Niños Benjamín Bloom, San Salvador, El Salvador. \\ ${ }^{*}$ Correspondencia \\ $\square$ wendyvenavides7@gmail.com \\ 1. (D) 0000-0003-3463-6800
}

Wendy María Lisseth Venavides Pineda ${ }^{1^{*}}$, Miriam Lourdes Dueñas de Chicas ${ }^{2}$, Guillermo Edgardo Barahona

Cavitating pulmonary tuberculosis an unusual clinical presentation in pediatric

\section{Citación recomendada:} Venavides Pineda WML,

Dueñas de Chicas ML, Barahona Escobar GE.

Tuberculosis pulmona cavitaria una presentación inusual en pediatría.

Alerta. 2021:4(3):114-118.

DOI: $10.5377 /$ alerta.v4i3.10907

\section{Recibido:}

23 de febrero de 2021

\section{Aceptado:}

13 de julio de 2021

\section{Publicado:}

26 de julio de 2021

\section{Contribución de autoría:}

WMVP': redacción del manuscrito. MLDDC ${ }^{2}, \mathrm{GEBE}^{3}$ : análisis de los datos y redacción del manuscrito.

\section{Conflicto de intereses:}

Los autores declaran no tener ningún tipo de conflicto de interés.

\begin{abstract}
Resumen
Se presenta el caso de una paciente femenina de 5 años quien fue ingresada en el Hospital Nacional de Niños «Benjamín Bloom», por presentar tos y fiebre persistente de 3 semanas. Previo al ingreso en el centro hospitalario, la paciente cursó con gastroenteritis aguda, por lo que en el Hospital Nacional Zacamil se sospechó fiebre tifoidea. Sin embargo, al continuar febril y con tos fue transferida al hospital. Durante el ingreso hospitalario se realizó el estudio para confirmar enfermedad tuberculosa presentando: prueba mantoux positiva (13 milímetros), radiografía de tórax con cavitación apical izquierda, baciloscopías positivas (16 puntos de los criterios de Stegen y Toledo). También se realizó una tomografía axial torácica computarizada que mostró lesión cavitada a nivel del lóbulo superior izquierdo, segmento apical posterior de paredes gruesas tabicadas. Iniciando tratamiento con 4 fármacos antituberculosos (isoniacida, rifampicina, pirazinamida y etambutol) y posterior al egreso hospitalario la paciente continuó con el tratamiento durante seis meses, presentando evolución clínica satisfactoria.

Palabras clave

Tuberculosis, cavitación, antituberculosos.
\end{abstract}

\section{Introducción}

La tuberculosis pulmonar es una infección común distribuida mundialmente con alta prevalencia en países en vías de desarrollo. Mycobacterium tuberculosis ha sido causante de muerte y enfermedad en la humanidad desde la era clásica y actualmente continúa siendo un problema médico y social con gran morbilidad y mortalidad' ${ }^{1}$. Se estima que durante el periodo comprendido entre los años 2000 al 2016, el diagnóstico oportuno y tratamiento eficaz salvaron alrededor de 53 millones de vidas y pese a ello la tuberculosis sigue siendo una gran amenaza en el mundo, ya que el $16 \%$ de los casos fallecen a causa de esta enfermedad. 
Un tercio de la población mundial está infectada con Mycobacterium tuberculosis; del 6-10\% de casos nuevos son en niños y cerca de 80000 niños fallecen cada año debido a esta infección ${ }^{2}$. La Organización Mundial de la Salud, para el año 2018, estima que 1,1 millones de casos nuevos de tuberculosis en la población pediátrica por debajo de los 14 años, alrededor de estos el 55 \% fueron subdiagnosticados o no reportados al inicio de la enfermedad, los pacientes pediátricos por debajo de los 5 años el porcentaje de subdiagnóstico asciende al $69 \%{ }^{3}$.

En El Salvador, la tuberculosis representa una amenaza para la población en general. En el año 2013 se reportaron 2176 casos de tuberculosis de todas las formas, con una tasa de 34,6 x 100000 habitantes, con un elevado índice desde el año 2011 respecto a 2008 (tasa de $28 \times 100000$ habitantes). Según el plan estratégico multisectorial para el control de la tuberculosis en El Salvador, diseñado en el año 2015 y que fue implementado entre los años 2016-2020, la incidencia de tuberculosis pulmonar en los niños menores de 14 años fue 0,9-1,1 por cada 100000 habitantes $^{4}$, reflejando que en pediatría la forma pulmonar de la enfermedad es menos frecuente en comparación con las formas extrapulmonares.

La tuberculosis primaria es común en la infancia con un curso subagudo y asociado con enfermedad extrapulmonar debido a la diseminación del bacilo por medio del torrente sanguíneo ${ }^{5}$. Es importante reconocer las diversas presentaciones clínicas y radiográficas de la tuberculosis primaria y posprimaria. Entre las formas de presentación de tuberculosis primaria en pediatría se encuentran: tuberculosis miliar, pleuritis exudativa y traqueobronquial y a medida que avanza la ciencia se descubren nuevas formas de tuberculosis similares a la del adulto. La cavitación es una manifestación rara de la tuberculosis primaria en lactantes y en niños que viven en comunidades expuestas al bacilo tuberculoso ${ }^{6}$. Actualmente hay pocos reportes de caso de esta presentación de la enfermedad en la edad pediátrica. A continuación, se presenta el caso de una paciente pediátrica que desarrollo tuberculosis pulmonar con cavitaciones.

\section{Presentación del caso}

Se trata de una paciente femenina de cinco años de edad, originaria del municipio de Ciudad Delgado, departamento de San Salvador, nivel socioeconómico medio, con antecedente de dos años previos. Al inicio de los síntomas la paciente convivía con un familiar con diagnóstico de tuberculo- sis pulmonar, que completó el tratamiento antituberculoso. Consultó en la unidad de emergencia del Hospital Nacional Zacamil, con historia de veinte días de fiebre de predominio nocturno no cuantificada de fuerte intensidad, que se acompañaba de escalofríos, diaforesis, adinamia e hiporexia y nueve días de diarrea, se decidió su ingreso con diagnóstico de gastroenteritis aguda. Durante su estancia hospitalaria persistió febril y se establece la sospecha diagnóstica de fiebre tifoidea. Por la persistencia de la tos, se realiza la toma de una radiografía de tórax, la cual mostró radiopacidad en vértice izquierdo y se refirió al hospital de tercer nivel.

En la unidad de emergencia Hospital Nacional de Niños «Benjamín Bloom» refirió historia de tres meses de tos con expectoración de predominio nocturno, sin producir emesis, ni cianosis, con el antecedente de haber consultado en múltiples ocasiones en establecimientos de primer nivel de atención, donde fue tratada con antitusivos, mucolíticos y agonistas $\beta$, adrenérgicos de acción corta, sin presentar mejoría. Al examen físico, se presentó agudamente enferma, pálida, hipoactiva, con temperatura de $39^{\circ} \mathrm{C}$, el resto de sus constantes vitales fueron normales. En la revisión por sistemas a nivel pulmonar se observaron tirajes subcostales e intercostales leves y estertores difusos en campo pulmonar izquierdo.

Los exámenes de laboratorio mostraron un conteo de $17600 \mathrm{~mm}^{3}$ glóbulos blancos, neutrófilos $62 \%$, linfocitos $27 \%$, proteína $C$ reactiva de $17,1 \mathrm{mg} / \mathrm{dl}$ y velocidad sedimentación globular $20 \mathrm{~mm} / \mathrm{hr}$; el resto de la química sanguínea fue normal. Se realizó la prueba cutánea de derivado proteico purificado, midiendo $13 \mathrm{~mm}$ a las 72 horas de lectura; ELISA para VIH fue negativo. Se realizaron tres baciloscopias con tinción de Ziehl-Neelsen que fueron positivas $(+++)$; no se cuantificaron niveles serológicos de inmunoglobulinas. La radiografía de tórax mostró radiopacidades apical e hiliar izquierda y sobre el vértice izquierdo una cavitación de paredes gruesas (16 puntos de los criterios de Stegen y Toledo, Tabla 1). Se realizó una tomografía axial computarizada torácica (TAC), que reportó lesión cavitada a nivel del lóbulo superior izquierdo, segmento apical posterior de paredes gruesas tabicadas compatible con tuberculosis pulmonar cavitada, como se muestra en la Figura 1 y Figura 2, iniciándose tratamiento antituberculoso con 4 fármacos de primera línea (isoniacida $5 \mathrm{mg}$ por kg, rifampicina $10 \mathrm{mg}$ por kg, pirazinamida $25 \mathrm{mg}$ por $\mathrm{kg}$ y etambutol $20 \mathrm{mg}$ por kg). Fue dada de alta y continuó manejo ambulatorio. Seis meses después la paciente finalizó 
el manejo con antituberculosos presentando evolución clínica satisfactoria.

\section{Discusión}

La tuberculosis es un gran reto para la salud pública en El Salvador como a nivel mundial. Representa un gran impacto debido a la morbilidad y mortalidad que ocasiona. Según la evidencia clínica, se espera que en pediatría predominen las formas clínicas extrapulmonares. La tuberculosis cavitaria ocurre principalmente entre el 40 al $87 \%$ descrita en adultos ${ }^{6}$ de la tuberculosis pulmonar; en la población pediátrica, esta proporción cambia con una tendencia menos frecuente durante la infancia y va en aumento en la adolescencia. En pediatría, la tuberculosis cavitaria es infrecuente. Existen reportes de casos en edades que en su mayoría comprenden la adolescencia y grupos etarios posteriores, siendo una de las principales razones por la que se reportó este caso. C. Perdikogianni et al. en el 2019 describieron dos casos de tuberculosis pulmonar cavitaria en niños inmigrantes en Creta, Grecia; uno en una paciente de 11 años de edad inmunocompetente y el otro en un paciente de 8 años de edad con inmunocompromiso (leucemia linfoblástica aguda). En ambos se obtuvo una evolución satisfactoria al instaurar tratamiento con antituberculosos, concluyendo que las formas severas de tuberculosis también pueden presentarse en niños, especialmente en aquellos con condiciones sociales de riesgo o inmunocompromiso ${ }^{8}$.

Cada vez se describen mecanismos más específicos que explican la evolución de Micobacterium tuberculosis y su adaptación al sistema inmunológico por múltiples vías. Existen diversas características inmunopatológicas que ayudan a entender cómo la tuberculosis dirige daños en la función pulmonar, cavitaciones, bronquiectasias y fibrosis.

Actualmente, existe una amplia evidencia científica que explica cómo la respuesta inflamatoria del huésped hacia la infección tuberculosa promueve el desarrollo de las cavitaciones. Clásicamente se ha descrito que la formación de la cavitación resulta de licuefacción del casium y es considerada el foco central de contagio y persistencia de la enfermedad ${ }^{8}$. También se han propuesto teorías alternativas controversiales en las que se atribuye un papel importante a la autoinmunidad, postulando que las micobacterias inducen respuestas inapropiadas en el huésped hacia los autoantígenos. Además, en la última década ya se han reportado errores innatos de la inmunidad asociados con formas graves o cursos atípicos de tuberculosis en la infancia.

Tabla 1. Criterios de Stegen modificados por Toledo7

\begin{tabular}{llll}
\hline Criterios & Puntaje establecido* & Hallazgos clínicos & Puntaje del paciente \\
\hline Baciloscopía & 7 puntos & Positiva $(+++)$ & 7 puntos \\
PPD & 3 puntos & $13 \mathrm{~mm}$ & 3 puntos \\
Radiografía de tórax & 2 puntos & Cavitación apical izquierda & 2 puntos \\
Cuadro clínico sugestivo & 2 puntos & + & 2 puntos \\
Antecedente & 2 puntos & + & 2 puntos \\
epidemiológico de & & & \\
Tuberculosis & & - & 0 puntos \\
Granuloma específico & 4 puntos & & 16 puntos \\
Puntaje & 20 puntos &
\end{tabular}

*Siete o más puntos confirmados permite un diagnóstico de certero.

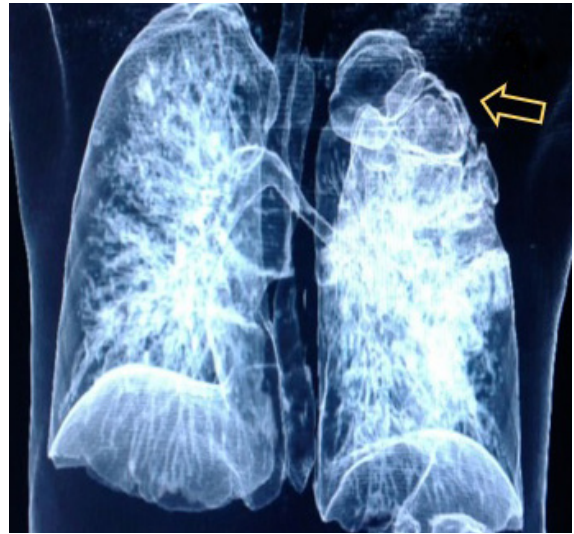

Figura 1. TAC pulmonar en $3 D$.

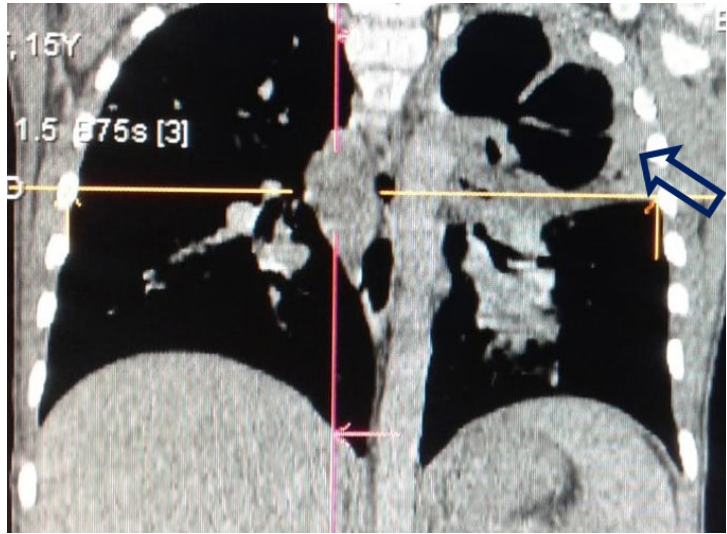

Figura 2. TAC pulmonar simple 
La cavitación pulmonar se caracteriza por la degradación de matriz celular por metalopeptidasas proteolíticas de matriz ${ }^{9,10}$ (MMP), encontradas con gran abundancia en los neutrófilos del parénquima pulmonar sugiriendo que la afluencia neutrofílica temprana conduce a un daño tisular que favorece la cavitación ${ }^{10}$. Los pacientes con cavitaciones tienen una carga bacteriana de hasta 10 bacilos $^{11}$, siendo altamente contagiosos $^{12}$. La mayoría de recaídas y fallas del tratamiento provienen de la tuberculosis cavitaria, siendo su diagnóstico crucial para la instauración de una terapia antituberculosa oportuna. Numerosos estudios han reportado la importancia de factores poblacionales, nutricionales, inmunológicos y otros que, en conjunto, desempeñan un rol protagónico en la morbilidad y mortalidad de pacientes con tuberculosis pulmonar cavitaria ${ }^{11-16}$.

Los estudios de imagen utilizados para el diagnóstico de tuberculosis pulmonar son bien conocido el rol que juega la radiografía de tórax; siendo parte del primer abordaje diagnóstico. La radiografía de tórax es comúnmente utilizada para visualizar el daño pulmonar y los hallazgos radiológicos distinguen entre la tuberculosis primaria y posprimaria ${ }^{17}$. Además, se destaca que el TAC es más sensible que la radiografía de tórax ${ }^{18}$. En este caso se realizaron ambos estudios y junto con el resto de herramientas diagnósticas se lograron establecer los criterios diagnósticos de enfermedad tuberculosa cavitaria e instaurar el tratamiento de manera pronta y exitosa, obteniendo un gran impacto en la evolución satisfactoria de la paciente ${ }^{18}$.

El daño pulmonar que produce Micobacterium tuberculosis ha sido estudiado en muchas perspectivas en una reciente publicación en la que Stek et al. describieron que el tratamiento convencional, junto con otras terapias adyuvantes, tiene un alto impacto en la reducción de lesiones pulmonares a largo plazo; entendiendo que la patogénesis del daño pulmonar es fundamental para tomar decisiones exitosas en la terapéutica y mejorar el pronóstico del paciente ${ }^{19-20}$.

\section{Conclusión}

La tuberculosis pulmonar es endémica en El Salvador. Excepcionalmente se observan casos de tuberculosis pulmonar cavitaria en niños menores de cinco años, siendo más común en la edad adulta, lo que resalta la importancia de este reporte de caso.

En la población pediátrica se deben hacer estudio de la enfermedad tuberculosa, principalmente cuando los niños son contactos de pacientes tuberculosos positivos.
La paciente no presentó los factores de riesgos clásicos descritos para el desarrollo de la enfermedad cavitaria. Se valoró que existió diagnóstico oportuno, ya que tuvo una evolución satisfactoria con el tratamiento antituberculoso.

\section{Agradecimiento}

A la contribución por parte de la Dra. Karla Escoto y Dra. Alejandra Baiza.

\section{Financiamiento}

Fondos propios de cada autor.

\section{Referencias bibliográficas}

1. Veedu PT, Bhalla AS, Vishnubhatla S, et al. Pediatric vs adult pulmonary tuberculosis: A retrospective computed tomography study. World J Clin Pediatr. 2013;2(4):70-76. DOI: 10.5409/wjcp.v2.i4.70

2. Sensoy G, Ceyhan M, Belet N, Karli A. 587 Cavitating Pulmonary Tuberculosis in Children. Arch. Dis. Child. 2012;97(Suppl 2):A170-A170. DOI: 10.1136/ archdischild-2012-302724.0587

3. Tahan TT, Gabardo BMA, Rossoni AMO. Tuberculosis in childhood and adolescence: a view from different perspectives. J Pediatr (Rio J). 2020;96(Suppl 1):99-110. DOI: 10.1016/j.jped.2019.11.002

4. Plan Estratégico Nacional Multisectorial para el control de la Tuberculosis en El Salvador 2016 - 2020. Ministerio de Salud. San Salvador, El Salvador. 2015. Disponible en: https://www.transparencia.gob.sv/system/ documents/documents/000/112/423/ original/plan estrategico multisectorial tb el salvador 2016 2020\%284\%29. pdf?1500371081

5. Boisson-Dupuis S, Bustamante J, ElBaghdadi J, Camcioglu Y, Parvaneh Safaa EA, Agader A, et al. Inherited and acquired immunodeficiencies underlying tuberculosis in childhood. Immunol Rev. 2015;264(1):103-120. DOI: 10.1111/imr.12272

6. Castiñeira Estévez A, López Pedreira MR, Pena Rodríguez MJ, Liñares Iglesias M. Manifestaciones radiológicas de la tuberculosis pulmonar. Med Integral 2002;39(5):192-206. Disponible en: https://www.elsevier.es/es-revistamedicina-integral-63-articulomanifestaciones-radiologicas-tuberculosispulmonar-13029945

7. Toledo A, Katz F, Montiel J, et-al. Criterios de diagnóstico en tuberculosis infantil. Rev. Mex. Pediatr 1979; 46: 236-239 
8. Perdikogianni C, Raissaki M, Christidou A, Galanakis E. Cavitary tuberculosis reemerging in immigrant children. Respir Med Case Rep. 2019;26:209-211. DOI: 10.1016/j. rmcr.2019.01.018.

9. Ong CW, Elkington PT, Friedland JS. Tuberculosis, pulmonary cavitation, and matrix metalloproteinases. Am J Respir Crit Care Med. 2014;190(1): 9-18. DOI: 10.1164/ rccm.201311-2106PP

10. Ordonez AA, Pokkali S, Sanchez-Bautista J, Klunk MH, Urbanowski ME, Kübler A, et al. Matrix Metalloproteinase Inhibition in a Murine Model of Cavitary Tuberculosis Paradoxically Worsens Pathology. J Infect Dis. 2019;219(4):633-636. DOI: 10.1093/ infdis/jiy373

11. Palaci M, Dietze R, Hadad DJ, Correa Ribeiro FK, Peres RL, Vinhas SA, et al. Cavitary disease and quantitative sputum bacillary load in cases of pulmonary tuberculosis. J Clin Microbiol. 2007;45(12):4064-4066. DOI: 10.1128/JCM.01780-07

12. Nakao M, Muramatsu $H$, Arakawa S, Sakai Y, Suzuki Y, Fujita K, et al. Immunonutritional status and pulmonary cavitation in patients with tuberculosis: A revisit with an assessment of neutrophil/lymphocyte ratio. Respir Investig. 2019;57(1):60-66. DOI: 10.1016/j.resinv.2018.08.007

13. Chao WC, Yen $\mathrm{CL}$, Wu CH, Shieh CC. How mycobacteria take advantage of the weakness in human immune system in the modern world. J Microbiol Immunol Infect. 2020;53(2):209-215. DOI: 10.1016/j. jmii.2019.10.008

14. du Preez K, Schaaf HS, Dunbar R, Walters E, Swartz A, Solomons R, et al. Complementary surveillance strategies are needed to better characterise the epidemiology, care pathways and treatment outcomes of tuberculosis in children. BMC Public Health. 2018;18(1):397. DOl: 10.1186/s12889-0185252-9

15. Nahid P, Dorman SE, Alipanah N, Barry PM, Brozek JL, Cattamanchi A, et al. Official American Thoracic Society/Centers for Disease Control and Prevention/Infectious Diseases Society of America Clinical Practice Guidelines: Treatment of Drug-Susceptible Tuberculosis. Clin Infect Dis. 2016;63(7): e147-195. DOl: 10.1093/cid/ciw376

16. George A, Andronikou S, Pillay T, Goussard P, Zar HJ. Intrathoracic tuberculous lymphadenopathy in children: a guide to chest radiography. Pediatr Radiol. 2017:47(10):1277-1282. DOI: 10.1007/ s00247-017-3890-1

17. Roya-Pabon CL, Perez-Velez CM. Tuberculosis exposure, infection and disease in children: a systematic diagnostic approach. Pneumonia (Nathan). 2016;8(1):23. DOI: 10.1186/s41479016-0023-9

18. Urbanowski ME, Ordonez AA, Ruiz-Bedoya CA, Jain SK, Bishai WR. Cavitary tuberculosis: the gateway of disease transmission. Lancet Infect Dis. 2020;20(6):e117-128. DOI: 10.1016/S1473-3099(20)30148-1

19. Moores RC, Brilha S, Schutgens F, Elkington PT, Friedland JS. Epigenetic Regulation of Matrix Metalloproteinase-1 and -3 Expression in Mycobacterium tuberculosis Infection. Front Immunol. 2017;8:602. DOI: 10.3389/fimmu.2017.00602

20. Stek C, Allwood B, Walker NF, Wilkinson RJ, Lynen L, Meintjes G. The Immune Mechanisms of Lung Parenchymal Damage in Tuberculosis and the Role of Host-Directed Therapy. Front Microbiol. 2018;9:2603. DOI: 10.3389/fmicb.2018.02603 